\title{
AN ADAPTIVE MORPHOLOGICAL MEAN FILTER FOR VERY HIGH-RESOLUTION REMOTE SENSING IMAGE PROCESSING
}

\author{
ZhiYong $\mathrm{Lv}^{\mathrm{a}}$ *, JunFei Shi ${ }^{\mathrm{a}}$, YingHui Wang ${ }^{\mathrm{a}}$ \\ ${ }^{a}$ School of Computer Science and Engineering, Xi'an University of Technology, Xi'an 710048, China. \\ Email:Lvzhiyong_fly@hotmail.com.
}

\author{
Commission IV, WG IV/3
}

KEY WORDS: Very high-resolution imagery, image filter, classification, support vector machine (SVM).

\begin{abstract}
:
Very high resolution (VHR) remote sensing imagery can reveal the ground object in greater detail, depicting their color, shape, size and structure. However, VHR also leads much original noise in spectra, and this original noise may reduce the reliability of the classification's result. This paper presents an Adaptive Morphological Mean Filter (AMMF) for smoothing the original noise of VHR imagery and improving the classification's performance. AMMF is a shape-adaptive filter which is constructed by detecting gradually the spectral similarity between a kernel-anchored pixel and its contextual pixels through an extension-detector with 8neighbouring pixels, and the spectral value of the kernel-anchored pixel is instead by the mean of group pixels within the adaptive region. The classification maps based on the AMMF are compared with the classification of VHR images based on the homologous filter processing, such as Mean Filter (MF) and Median Filter(MedF). The experimental results suggest the following: 1) VHR image processed using AMMF can not only preserve the detail information among inter-classes but also smooth the noise within intra-class; 2) The proposed AMMF processing can improve the classification's performance of VHR image, and it obtains a better visual performance and accuracy while comparing with MF and MedF.
\end{abstract}

* Corresponding author 


\section{INTRODUCTION}

In recent decades, a lot of remote sensing images with very high resolution (VHR) are available in the urban area. Since VHR image can depict the grounded object information, such as shape, structure, size, and texture, in detail, these images open new perspectives for remote sensing application in urban monitoring, environmental assessment, decision-making, et al especially, lots of these applications are depended on the result of ground classification.

However, it is well known that the higher resolution image does not naturally mean the higher classification accuracies (Wilkinson 2005, Huang and Zhang 2013). On the one hand, VHR image increases the correlation among pixels, and this results in a relevant amount of spatial features in VHR image, these features are useful for visual interpretation and classification(Wang, Dai et al. 2012). On the other hand, since the constraints of remote sensing technique, images with a very high resolution are typically limited to generally $3 \sim 4$ spectral bans(worldview-2 is 0.41 meter and 8-bands)(Gianinetto, Rusmini et al. 2014). In other words, high resolution and low spectral properties result in an increment of the intra-class variance and a decrease of the inter-class variance, which leads original spectral noise into the classifying thematic map and enhance the difficulty of separability (Carleer, Debeir et al. 2004, Blaschke 2010). Such as, Ouyang Zhang has shown that as the spatial resolution of remote sensing data increases, the original spectral noise of pixel-wise approach becomes more serious (Ouyang, Zhang et al. 2011). In fact, much effects, such as a sensor, spatial uncertain also bring noise into the VHR image, and a lot of pixels wise approaches are very sensitive to this noise(Soille 2003, Huang and Zhang 2008, Duro, Franklin et al. 2012).

To improve the performance of VHR image classification, many researchers have worked on object-oriented classification. The object-oriented approach generally starts with multi-scale image objects as the classifying unit for image analysis, instead of a single pixel(Baatz and Sch01pe 2000, Tang, Zhang et al. 2011, Li, Myint et al. 2014). Image objects are formed by grouping pixels together which are spectral similar and spatially contiguous, because of the classifying unit is raised from pixel to object(grouping pixels), the effect of noise can be alleviated by using object-oriented method and the accuracy of the classification can be further improved(Yu, Gong et al. 2006). For example, Myint et al. compared pixel-based and objectoriented classification of land cover using VHR image, the results have shown that object-oriented approach obtains higher accuracies and better visual performance than pixel-based classification(Myint, Gober et al. 2011); Ouyang Zhang presented a case study for mapping saltmarsh plants using VHR imagery, the results shown that object-based model significantly reduced the noise when compared with pixel-based approach(Ouyang, Zhang et al. 2011); As well, an experiment contained three pixel-based classifications which all produced a similarly speckled noise appearance comparing to object-based approach(Duro, Franklin et al. 2012). The Object-based approach could alleviate original noise in the result of VHR remote sensing image classification, and some advantages of object-oriented techniques in VHR imagery have been proved in (Pagot, Pesaresi et al. 2008, Agarwal, Vailshery et al. 2013, Blaschke, Hay et al. 2014). However, some limitations of object-based approach to VHR image classification relative to a pixel-based approach were also explored, such as segmental scale-parameters is difficult to obtain(Liu and Xia 2010): too large scale will smooth the detailed information(such as edge, texture) while removing noise and too small smaller scale can not cover the enough noise to improve the performance of classification.

Another family means for improving the accuracies of VHR image classification are spectral-spatial classification methods. Spatial information is inherited in the real world remote sensing image, especially in the VHR images(Li, Zang et al. 2014). Therefore, spatial information is employed to couple with spectra to make up spectral information's insufficient, results in smoothing noise and improving the accuracy of VHR image classification, e.g., Tarabalka et al. proposed a new spectralspatial classification scheme for hyperspectral images(Tarabalka, Benediktsson et al. 2009);Huang et al proposed a multi-feature model, including spectra, structure and semantic feature for VHR image classification(Huang and Zhang 2013); and Xia et al. proposed a new spatial-spectral classification method to enhance the performance of hyperspectral images by integrating rotation forests and Markov random fields(Xia, Chanussot et al. 2015). In addition, spatialspectral kernels have been investigated for classification of VHR image in many types of research, such as the morphological kernel and sparse kernel(Liu, Wu et al. 2013). To exploring the spatial information and improving the performance of VHR image classification, mathematical morphology was extended by increasing sizes of structuring element(Benediktsson, Pesaresi et al. 2003) and stacking different shapes of structuring element(Lv, Zhang et al. 2014). Several advances of spectral-spatial approach for the classification of hyperspectral images were summarized in the previous research(Fauvel, Tarabalka et al. 2013). However, spatial-spectral feature based approach relies on the performance of the spatial feature extracted technique. In order to get accurate spatial features, advanced feature extraction algorithms are usually required, but those processes may be time-consuming and experience depended.

Different from the object-oriented and spatial-spectral feature based classification approach described previously, image filter is also adopted to smooth the original noise of hyperspectral imagery with high resolution. Such as Dalla Mura et al., defined morphological attributes(APs) based on the sequential application of morphological attribute filters(Dalla Mura, Benediktsson et al. 2010), and these filters were also extended(Dalla02Mura, Atli02Benediktsson et al. 2010); Angelos et al. developed an image classification framework which integrated with nonlinear scale-space filter(Tzotsos, Argialas et al. 2011); Recently, edge-preserving filter has become a very hot topic of hyperspectral image processing and applied successfully in many applications(Kotwal and Chaudhuri 2010, Kang, Li et al. 2014). K. Tanaka et al. given a local subspace classifier by integrating Gabor filter decomposition(Tanaka and Hotta 2013). Sun et al. compared mean filter between and median filter algorithm(Huang, Yang et al. 1979) in image denoising field(Sun and Qu 2014).

Although the filter is a powerful tool for denoising noise from the image, the practical application of filters existed two problems. 1)Most of the filter was investigated on the hyperspectral image, and VHR image is more or less ignored; 2) many filters, such as mean filter(MF), a median filter(MF), are related to a regular window as filter's convolution. However, single size and the regular window may be unable to cover the multifarious spatial information in the entire image. Therefore, it should be noted that the existing filter is related to the spectral and regular domain alone, few filters refer to the joint spectralspatial domains, especially for the VHR remote sensing image classification. 
In this paper, an adaptive mean morphological filter (AMMF) is proposed for reducing the original noise and improving the performance of VHR image classification. Comparing with that of MF and MedF, the proposed filter runs through the whole VHR image by morphological convolution in an adaptive manner. To verify the performance of the proposed approach, experiments were designed on the base of three VHR images: 1)a VHR false colour image of Pavia university; 2) IKONOS data obtained from Reykjavik, Iceland; 3)a VHR false colour image of Pavia center. In these experiments, results of classification based on the proposed AMMF is compared with that of no filtering process, mean filter and median filter.

The rest of paper is organized as follows. Section-II describes the proposed approach of AMMF. Section-III shows the framework of the proposed AMMF applied in the classification of VHR image. Section IV details experiments on three VHR data sets. Section V presents the conclusion of this paper.

\section{NEW IMAGE DENOISING APPROACH OF AMMF FOR VHR IMAGE}

High resolution and low spectral characteristic of VHR image lead much original noise in the image, and this results in an unsatisfactory classification map. In this section, an image filter, called AMMF, is proposed for reducing the original noise of VHR image.

AMMF adds an extension of the mean filter. Unlike the single size and regular window of the mean filter, the shape and size of the convolution region of AMMF are extracted in a pixel-bypixel manner, wherein the region of each pixel has a higher homogeneous. The shape of an adaptive region represents the contextual feature surrounding a kernel-anchored pixel, the size of the adaptive region is constrained by two thresholds in spectral and spatial domains.

The algorithm of AMMF is shown in Figure1, and it will be detailed as three following steps: 1) Extension of AMMF's region; 2) Erosion-based operation within AMMF's region; 3 ) Calculate the mean value of labeled pixels.

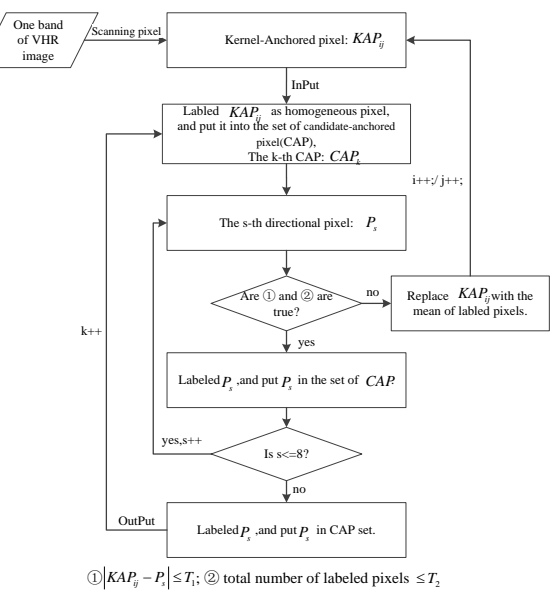

Figure 1. Flowchart of the proposed AMMF algorithm.

\subsection{The algorithm of region-extension for AMMF.}

An adaptive extension used to detect the contextual feature and the object's overall contour are symmetric for an image object (such as road, build et al). As it is shown in Figure1, if the surrounding pixel $P_{S}$ and $K A P_{s i j}$ met the condition-1, labelled
$P_{j}$ as homogeneous pixel and put it into the set of candidateanchored pixels (CAP). A pixel in the set of CAP is preparing as another level of the anchored pixel for the region extension in the spatial domain, 8-neighbouring pixels surrounding a pixel of CAP is compared with $K A P_{s i j}$ in spectral domain. Therefore, this promises all the labelled pixels are spectral homogeneous and the extension of AMMF's region is adaptive in the spatial domain. In other words, CAP is used to extend the adaptive region in a recursive way.

Based on the aforementioned algorithm, the extension of a homogeneous region for an AMMF will cease if either of the above conditions is not net. In this case, the extension of KAP will be terminated, the value of KAP is replaced with the mean of pixels within the adaptive region and the algorithm will skip to the (i+1)-th KAP. The whole image is run in this way and each pixel will be taken as once KAP.

\subsection{Erosion-based operation within AMMF's region.}

In practical application, when process VHR image using AMMF, since the spatial complexity of pixels or the spectral heterogeneity of the ground objects, the hollow contour of the region may be produced in the processing of an adaptive regionextension. Figure-2 illustrates three samples of VHR image of Pavia Centre, Italy. It can be found that the appendages of the building (such pump-house, chimney, et al, on the top of the building), and the sun' s height, all those will lead original noise in the VHR remote sensing image. Unfortunately, these noise affect obviously the extension of AMMF's region: if the current KAP is an objective pixel, fitted parameters $\left(T_{1}\right.$ and $\left.T_{2}\right)$ may result in hollow region of AMMF, such as the second column in Figure-2; On the contrary, if the KAP is a noise pixel, since the difference between noise-pixel and its CAP is over T1, and the extension ceased, see the third column in Figure-2. Ideally, all KAPs consisting an object has a similar shape and size of the adaptive region. In fact, the region of a noise-KAP is smaller than that of an objective KAP, and result in a larger contextual filter for an objective KAP.
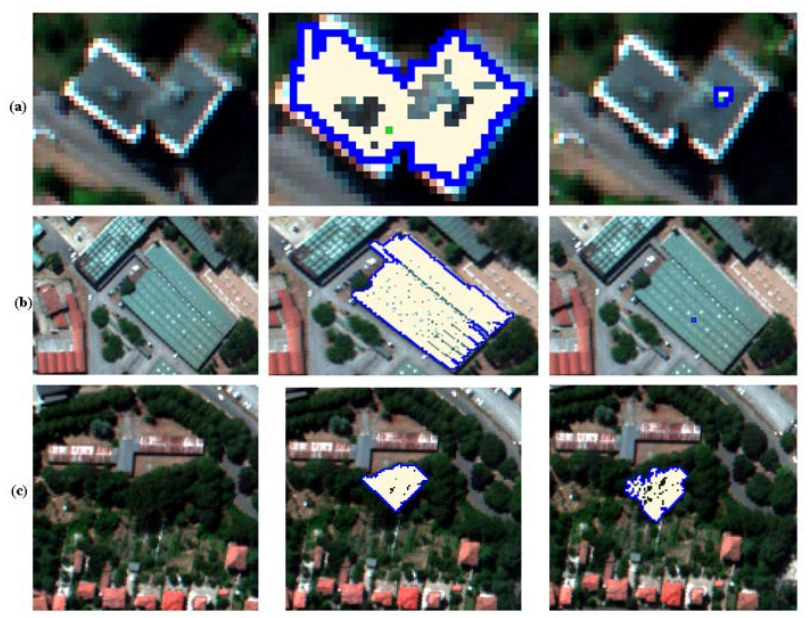

Figure 2. Examples of Extension for AMMF in different classes.

To enhance an objective KAP's filtering effect and weaken the influence of noise's KAP, an erosion-based strategy is adopted here. As well as the erosion operation of mathematical morphology, which tests whether a structuring element(SE) "fits" an object within a neighbourhood, that's means the erosion removes pixels from object's boundaries, and the number of pixels removed from an object depends on the size 
and shape of SE used to process the image. The erosion-based strategy used here is to remove the noise pixels inside from a homogeneous objective area, which is defined as

$$
E O\left(x_{i}\right)=\frac{1}{n} \cdot \sum_{l=0}^{n} v_{l}
$$

This formula represents the erosion operation to replace the hollow pixel within an adaptive region of AMMF. Where $E O\left(x_{i}\right)$ is defined as the erosion-value of noise pixel $x_{i}, v_{l}$ is the value of a pixel which is labelled in the extension of AMMF's region. $\mathrm{n}$ is the total number of the labelled pixel, the $E O\left(x_{i}\right)$ is eroded the mean value of labelled pixels. While the hollow pixels were eroded by the mean of the labeled pixel, and it is promised the pixels of AMMF's region have a higher homogeneous. In other words, while the hollow pixels within the region of an AMMF were filled, the adaptive region becomes a solid region, and all the labelled pixels consisted the adaptive solid-region are similar in terms of their spectral value.

\subsection{Calculate the mean value of labelled pixels}

After determining the adaptive region of AMMF, an AMMF is defined as

$$
\operatorname{AMMF}\left(x_{i}\right)=\frac{1}{L} \cdot \sum_{l=0}^{l=L} v_{l}
$$

Where $\operatorname{AMMF}\left(x_{i}\right)$ is the filtering process of the KAP: $X_{i}, \mathrm{~L}$ is the total number of the labelled pixel within the adaptive region and $v_{l}$ is the value of a labelled pixel. The value of a KAP filtering by its AMMF is equal to the average mean of the labelled pixels wherein the adaptive region.

It should be noted that AMMF differs from the traditional MF in the following aspects.

1) MF is simply to replace each pixel value in an image with the mean ('average') value of its neighbours. This has the effect of eliminating pixel values which are unrepresentative of their surroundings. Mean filter is usually thought of as a regular filter, such as $3 \times 3$ squares, $5 \times 5$ squares. However, AMMF filters an image by replacing each pixel value with the mean of each pixel's adaptive region. The aims of AMMF is to describe the overall contextual features and let all pixels of an object have the higher similarity in spectra.

2) MF yields to linear filters and it can remove noise, but it also smoothes the edges and boundaries, and may "erase" the details whose size is not equal to the window size. As a result, an image filtered by MF becomes "fuzzy"; however, AMMF is nonlinear in the spatial domain, the value of a pixel is filtered whether or not depends on the spectral differences between a kernel-anchored pixel and its contextual pixels. The extension of an AMMF's region is self-adaptive and constrained by two parameters: $T_{1}$ and $T_{2}$. Hence, AMMF not only smoothes the noise of intra-class, but retains the detail of inter-classes.

\section{EXPERIMENTS}

To illustrate the strength of AMMF, four VHR images are respectively filtered with AMMF and MF, the results are compared in Figure 3. It can be found that AMMF can smooth the building, trees and retain the edges between them. The similar observation can be found in Figure 4. To investigate the proposed AMMF more comprehensively, classification maps based on the proposed AMMF and the raw image without any filter processing shown in Figure 4, it is clear that the proposed
AMMF has the ability for reducing the noise of remote sensing image with high spatial resolution. In addition, it can preserve the boundary information between different targets while comparing with the tradition mean filter.

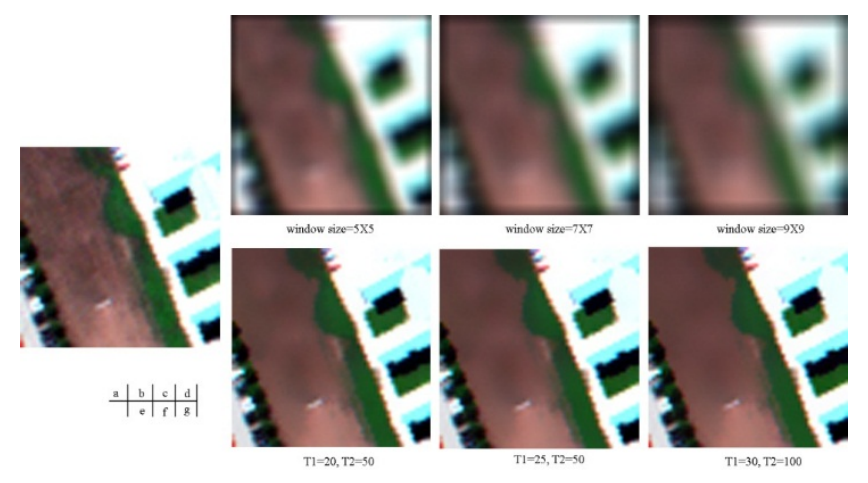

Figure 3. Filtering Result Comparison between MF and AMMF using a VHR sample image. (a) is a VHR image with 1.0-meter resolution and R-G-B three bands; b,c,d are the results process by MF with different window size; e, $\mathrm{f}$ and $\mathrm{g}$ are the results processed by AMMF with different constrained parameters: T1 and $\mathrm{T} 2$.

In addition, we investigated the classified performance of the proposed AMMF based classification by comparing the proposed AMMF with that of MF and MedF, including the original remote sensing the original VHR image. As it is shown in Figure-5, it can be seen that the proposed AMMF improve the performance of classification than that of MF and MedF or the original VHR image without any processing in all the experiments.
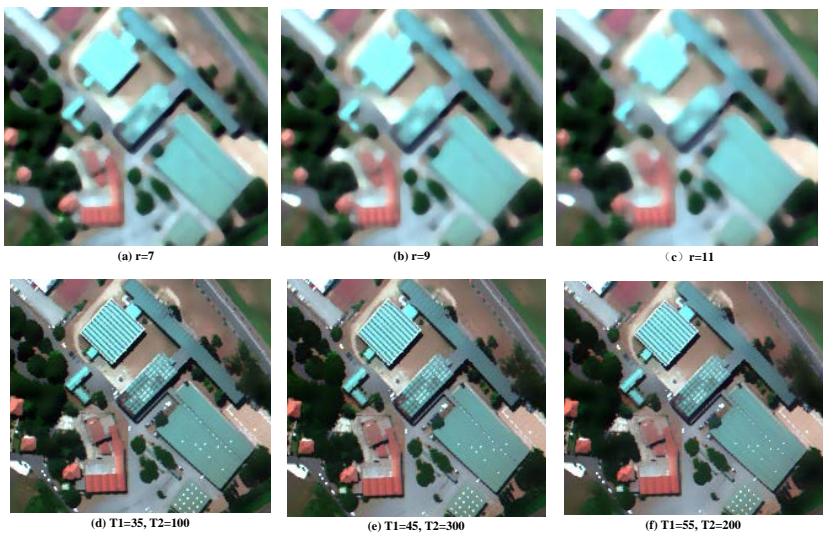

Figure 4. Visual performance comparison between the proposed AMMF processing and that of MedF filter: a to $\mathrm{c}$ are the filtered result using MedF with the $r=7, r=9$ and $r=11$; $d$ to $f$ are the results based on AMMF processing with the parameters' setting: $(\mathrm{T} 1=35, \mathrm{~T} 2=100),(\mathrm{T} 1=45, \mathrm{~T} 2=300)$, and $(\mathrm{T} 1=55$, $\mathrm{T} 2=200)$. 


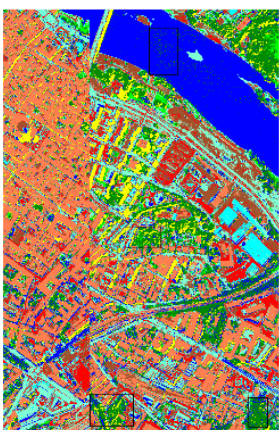

(a)

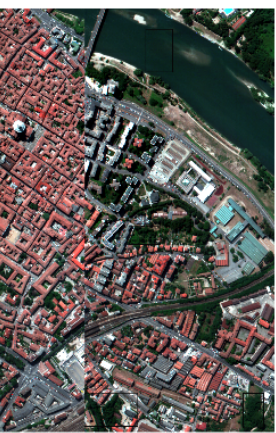

(b)

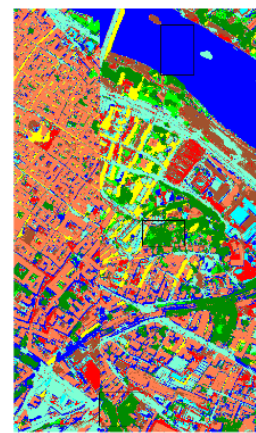

(c)
Figure 5. Visual comparison of the classification maps between the original Pavia centre image and the proposed AMMF-based classification through SVM. (a) The classification map based on the original image; (b) the original image-Pavia centre; (c) the classification map based on the processing of AMMF.

\section{CONCLUSION}

To conquer the noise of VHR remote sensing image, the proposed AMMF, which were adaptive by considering spatial contextual information and spectral similarity, and then extend gradually the region of AMMF using an extension- detector with 8-neighbouring pixels. Therefore, the AMMF not only have the ability to preserve the detail information in interclasses but also can smooth the noise in the intra-class. In other words, the filter of AMMF is dynamically adaptive according to with the shape, size, and spectral different of an objective object. As a topic of future research, another possible approach which is parameter (T1 and T2) automation can be considered. In theory, if $\mathrm{T} 1$ and $\mathrm{T} 2$ are acquired in a self-adaptive way by considering the spectral difference of contextual pixels, such an approach will be provided an image filter with a higher degree of automation, and the advantage of such an approach maybe more effective than that of the proposed approach for VHR image classification.

\section{ACKNOWLEDGMENT}

The authors would like to thanks the referenced reviewers for their helpful suggestions in improving this paper. This work is supported by National Natural Science Foundation of China (No. 61472319) and Shaanxi Science Research Plan (No.2015JZ015).

\section{REFERENCES}

Agarwal, S., L. S. Vailshery, M. Jaganmohan and H. Nagendra 2013. "Mapping urban tree species using very high resolution satellite imagery: comparing pixel-based and object-based approaches." ISPRS International Journal of Geo-Information 2(1): 220-236.

Baatz, M. and A. Sch01pe 2000. "2000: Multiresolution Segmentation - an optimization approach for high quality multiscale image segmentation." Angewandte Geographische Informationsverarbeitung XII.

Benediktsson, J. A., M. Pesaresi and K. Amason 2003. "Classification and feature extraction for remote sensing images from urban areas based on morphological transformations." IEEE Transactions on Geoscience \& Remote Sensing 41(9): 1940--1949.

Blaschke, T. 2010. "Object based image analysis for remote sensing." ISPRS journal of photogrammetry and remote sensing 65(1): 2-16.
Blaschke, T., G. J. Hay, M. Kelly, S. Lang, P. Hofmann, E. Addink, R. Q. Feitosa, F. van der Meer, H. van der Werff and F. van Coillie 2014. "Geographic object-based image analysistowards a new paradigm." ISPRS journal of photogrammetry and remote sensing 87: 180-191.

Carleer, A. P., O. Debeir and E. Wolff, 2004. Comparison Of Very High Spatial Resolution Satellite Image Segmentations. SPIE.

Dalla02Mura, M., J. Atli02Benediktsson, B. Waske and L. Bruzzone 2010. "Extended profiles with morphological attribute filters for the analysis of hyperspectral data." International Journal of Remote Sensing 31(22): 5975-5991.

Dalla Mura, M., J. A. Benediktsson, B. Waske and L. Bruzzone 2010. "Morphological Attribute Profiles for the Analysis of Very High Resolution Images." IEEE Transactions on Geoscience \& Remote Sensing 48(10): 3747 - 3762.

Duro, D. C., S. E. Franklin and M. G. Dubé 2012. "A comparison of pixel-based and object-based image analysis with selected machine learning algorithms for the classification of agricultural landscapes using SPOT-5 HRG imagery." Remote Sensing of Environment 118(6): 259-272.

Duro, D. C., S. E. Franklin and M. G. Dubé 2012. "A comparison of pixel-based and object-based image analysis with selected machine learning algorithms for the classification of agricultural landscapes using SPOT-5 HRG imagery." Remote Sensing of Environment 118: 259-272.

Fauvel, M., Y. Tarabalka, J. A. Benediktsson, J. Chanussot and J. C. Tilton 2013. "Advances in spectral-spatial classification of hyperspectral images." Proceedings of the IEEE 101(3): 652675.

Gianinetto, M., M. Rusmini, G. Candiani, G. D. Via, Federico, Frassy, A. Marchesi and L. Dini 2014. "Hierarchical classification of complex landscape with VHR pan-sharpened satellite data and OBIA techniques." European Journal of Remote Sensing 47.

Huang, T., G. Yang and G. Tang 1979. "A fast two-dimensional median filtering algorithm." Acoustics Speech \& Signal Processing IEEE Transactions on 27.

Huang, X. and L. Zhang 2008. "An Adaptive Mean-Shift Analysis Approach for Object Extraction and Classification From Urban Hyperspectral Imagery." IEEE Transactions on Geoscience \& Remote Sensing 46(12): 4173 - 4185.

Huang, X. and L. Zhang 2013. "An SVM Ensemble Approach Combining Spectral, Structural, and Semantic Features for the Classification of High-Resolution Remotely Sensed Imagery." IEEE Transactions on Geoscience \& Remote Sensing 51(1): $257-272$

Huang, X. and L. Zhang 2013. "An SVM ensemble approach combining spectral, structural, and semantic features for the classification of high-resolution remotely sensed imagery." Geoscience and Remote Sensing, IEEE Transactions on 51(1): 257-272.

Kang, X., S. Li and J. A. Benediktsson 2014. "Spectral-spatial hyperspectral image classification with edge-preserving 
filtering." Geoscience and Remote Sensing, IEEE Transactions on 52(5): 2666-2677.

Kotwal, K. and S. Chaudhuri 2010. "Visualization of Hyperspectral Images Using Bilateral Filtering." IEEE Transactions on Geoscience \& Remote Sensing 48(5): 2308 2316.

Li, M., S. Zang, B. Zhang, S. Li and C. Wu 2014. "A review of remote sensing image classification techniques: The role of spatio-contextual information." European Journal of Remote Sensing 47: 389-411.

Li, X., S. W. Myint, Y. Zhang, C. Galletti, X. Zhang and B. L. T. Ii 2014. "Object-based land-cover classification for metropolitan Phoenix, Arizona, using aerial photography." International Journal of Applied Earth Observation \& Geoinformation 33(12): 321-330.

Liu, D. and F. Xia 2010. "Assessing object-based classification: advantages and limitations." Remote Sensing Letters 1(4): 187194.

Liu, J., Z. Wu, Z. Wei, L. Xiao and L. Sun 2013. "SpatialSpectral Kernel Sparse Representation for Hyperspectral Image Classification." IEEE Journal of Selected Topics in Applied Earth Observations \& Remote Sensing 6(6): 2462 - 2471.

Lv, Z. Y., P. Zhang, J. A. Benediktsson and W. Z. Shi 2014. "Morphological Profiles Based on Differently Shaped Structuring Elements for Classification of Images With Very High Spatial Resolution." Selected Topics in Applied Earth Observations \& Remote Sensing IEEE Journal of 7.

Myint, S. W., P. Gober, A. Brazel, S. Grossman-Clarke and Q. Weng 2011. "Per-Pixel Vs. Object-Based Classification Of Urban Land Cover Extraction Using High Spatial Resolution Imagery." Remote Sensing of Environment 115(5): 1145-1161.

Ouyang, Z.-T., M.-Q. Zhang, X. Xie, Q. Shen, H.-Q. Guo and B. Zhao 2011. "A comparison of pixel-based and objectoriented approaches to VHR imagery for mapping saltmarsh plants." Ecological Informatics 6(2): 136-146.

Ouyang, Z. T., M. Q. Zhang, X. Xie, Q. Shen, H. Q. Guo and B. Zhao 2011. "A comparison of pixel-based and object-oriented approaches to VHR imagery for mapping saltmarsh plants." Ecological Informatics 6(2): 136-146.

Pagot, E., M. Pesaresi, D. B. Ehrlich and D. 2008. "Development of an object - oriented classification model using very high resolution satellite imagery for monitoring diamond mining activity." International Journal of Remote Sensing 29(2): 499-512.

Soille, P. 2003. "Morphological Image Analysis-Principles and Applications." Environment \& Planning B Planning \& Design 28(5): 800-801.

Sun, X. X. and W. Qu 2014. "Comparison between Mean Filter and Median Filter Algorithm in Image Denoising Field." Applied Mechanics \& Materials 644-650.

Tanaka, K. and S. Hotta ,2013,. Local Subspace Classifier with Gabor Filter Decomposition for Image Classification. Pattern Recognition (ACPR), 2013 2nd IAPR Asian Conference on.
Tang, Y., L. Zhang and X. Huang 2011. "Object-oriented change detection based on the Kolmogorov-Smirnov test using high-resolution multispectral imagery." International Journal of Remote Sensing 32: 5719-5740.

Tarabalka, Y., J. A. Benediktsson and J. Chanussot 2009. "Spectral-spatial classification of hyperspectral imagery based on partitional clustering techniques." Geoscience and Remote Sensing, IEEE Transactions on 47(8): 2973-2987.

Tzotsos, A., D. Argialas and K. Karantzalos 2011. "Objectbased image analysis through nonlinear scale-space filtering." Isprs Journal of Photogrammetry \& Remote Sensing 66(1): 216.

Wang, L., Q. Dai, L. Hong and G. Liu 2012. "Adaptive regional feature extraction for very high spatial resolution image classification." Journal of Applied Remote Sensing 6(4): 339355.

Wilkinson, G. G. 2005. "Results and implications of a study of fifteen years of satellite image classification experiments." IEEE Transactions on Geoscience \& Remote Sensing 43(3): $433-440$.

Xia, J., J. Chanussot, P. Du and X. He 2015. "Spectral-Spatial Classification for Hyperspectral Data Using Rotation Forests With Local Feature Extraction and Markov Random Fields." Geoscience and Remote Sensing, IEEE Transactions on 53(5): 2532-2546.

Yu, Q., P. Gong, N. Clinton, G. Biging, M. Kelly and D. Schirokauer 2006. "Object-based detailed vegetation classification with airborne high spatial resolution remote sensing imagery." Photogrammetric Engineering \& Remote Sensing 72(7): 799-811. 